\title{
Relationship between Sea Surface Drag Coefficient and Wave State
}

\author{
Jian Shi, Zhihao Feng, Yuan Sun *, Xueyan Zhang, Wenjing Zhang and Yi Yu \\ College of Meteorology and Oceanography, National University of Defense Technology, Changsha 410073, China; \\ shijian@nudt.edu.cn (J.S.); fengzhihao17@nudt.edu.cn (Z.F.); xyzhang_nj@nudt.edu.cn (X.Z.); \\ zhangwenjing21@nudt.edu.cn (W.Z.); yi.yu@nudt.edu.cn (Y.Y.) \\ * Correspondence: sunyuan17a@nudt.edu.cn
}

Citation: Shi, J.; Feng, Z.; Sun, Y.; Zhang, X.; Zhang, W.; Yu, Y. Relationship between Sea Surface Drag Coefficient and Wave State. J. Mar. Sci. Eng. 2021, 9, 1248 . https://doi.org/10.3390/jmse9111248

Academic Editor: Luca Martinelli

Received: 28 September 2021

Accepted: 2 November 2021

Published: 10 November 2021

Publisher's Note: MDPI stays neutral with regard to jurisdictional claims in published maps and institutional affiliations.

Copyright: (c) 2021 by the authors. Licensee MDPI, Basel, Switzerland. This article is an open access article distributed under the terms and conditions of the Creative Commons Attribution (CC BY) license (https:// creativecommons.org/licenses/by/ $4.0 /)$.

\begin{abstract}
The sea surface drag coefficient plays an important role in momentum transmission between the atmosphere and the ocean, which is affected by ocean waves. The total air-sea momentum flux consists of effective momentum flux and sea spray momentum flux. Sea spray momentum flux involves sea surface drag, which is largely affected by the ocean wave state. Under strong winds, the sea surface drag coefficient $\left(C_{D}\right)$ does not increase linearly with the increasing wind speed, namely, the increase of $C_{D}$ is inhibited by strong winds. In this study, a sea surface drag coefficient is constructed that can be applied to the calculation of the air-sea momentum flux under high wind speed. The sea surface drag coefficient also considers the influence of wave state and sea spray droplets generated by wave breaking. Specially, the wave-dependent sea spray generation function is employed to calculate sea spray momentum flux. This facilitates the analysis not only on the sensitivity of the sea spray momentum flux to wave age, but also on the effect of wave state on the effective $C_{D}\left(C_{D, \text { eff }}\right)$ under strong winds. Our results indicate that wave age plays an important role in determining $C_{D}$. When the wave age is $>0.4, C_{D}$ decreases with the wave age. However, when the wave age is $\leq 0.4, C_{D}$ increases with the wave age at low and moderate wind speeds but tends to decrease with the wave age at high wind speeds.
\end{abstract}

Keywords: sea surface drag coefficient; wave state; wave age; sea spray droplets

\section{Introduction}

Sea surface wind stress is the main factor that drives upper-ocean circulation and wind waves. An accurate estimate of sea surface wind stress is important for modelling and predicting the dynamic processes involving ocean and atmosphere interactions. Therefore, sea surface wind stress has been widely observed and studied in the past decades, which is usually parameterized by a sea surface drag coefficient $\left(C_{D}\right)$ or sea surface aerodynamic roughness [1-4]. The study of sea surface wind stress is thus the study of $C_{D}$ or sea surface aerodynamic roughness.

The initial atmospheric boundary layer theory assumed that the interface was the fixed land. Therefore, under the condition of neutral stable stratification, air-sea flux only depended on the variation of wind speed, and the influence of wind waves was not taken into account. Researchers tended to parameterize $C_{D}$ only by wind speed [5-8]. However, these relationships between $C_{D}$ and wind speed obtained by different researchers differed greatly.

Ocean waves often exist on the sea surface, and their simulations and effects have been studied extensively $[9,10]$. In recent years, more and more evidence has indicated that the influence of wave state on wind stress cannot be ignored when investigating air-sea interaction [4,11-13]. However, the results of laboratory and field measurements were not always consistent, and the relationships between $C_{D}$ and the wave age obtained by the two approaches were even contradictory $[11,14]$. 
Different results were obtained using the parameterization of $C_{D}$ with different wave state parameters. Guan and Xie [3] pointed out that the drag coefficient was a linear function of wind speed, and its slope was related to the wave steepness parameter $\delta$. Shi et al. [13] analyzed the correlations between four wave parameters and $C_{D}$ and found that the windsea Reynolds number $R_{B}$ had the best parameterization effect. Zhao and $\mathrm{Li}$ [4] used laboratory and offshore observation data to compare the relationships between several drag coefficients and wind wave parameters; they pointed out that wave age and wave steepness had their own unique effects on air-sea flux.

Water depth also affects the drag coefficient, and the roughness of the sea surface is higher than that in the open ocean for corresponding values of wind speed [15-17]. Furthermore, young waves are rougher than older ones, and the limited fetch in semienclosed tidal basins limits the presence of old waves in favor of young ones [17].

The researchers generally use the tropical cyclone (TC) wind field model or meso-scale atmospheric model to drive the numerical simulation of the drag coefficient, and they pointed out that the simulated drag coefficient and roughness contribute remarkably to the simulated TC track, intensity, and wave [18-20]. With the deepening of observational studies, researchers found that $C_{D}$ did not increase linearly under high wind speed conditions. Moreover, when the wind speed was greater than a certain value, the increase of $C_{D}$ was inhibited and even attenuated [21,22]. Laboratory observations also indicated this phenomenon $[23,24]$. Takagaki et al. [24] found a strong correlation between $C_{D}$ and wave spectrum energy in the laboratory. Zhao and Li [4] proposed a method of double parameters to parameterize $C_{D}$ and pointed out that the drag coefficient changed with wave age and wave steepness independently. Specifically, at high wind speeds, due to strong wave breaking, the wave steepness reached its peak and remained stable, while the wave age continued to decrease. Therefore, it was found that the change of the drag coefficient with the wave age was not monotonous, showing two distinct variations when the wave age was greater or less than 0.4 .

At high wind speed, the waves will break, and the breaking waves produce sea spray droplets. Sea spray droplets change the state of the air-sea interface and then affect various physical processes at the air-sea interface. According to previous analysis, droplets weaken $C_{D}$ at high wind, but the phenomenon's physical mechanism is controversial [25,26]. Makin [26] regarded droplets as suspended particles. In the suspended layer, the concentration of droplets forms a stable layer, called the droplets boundary layer, which prevents turbulence from approaching the sea surface, thus attenuating $C_{D}$ under high wind speed.

Andreas [25] proposed an alternative explanation, which is that the sea spray momentum flux weakens $C_{D}$ at high wind speeds. As droplets enter the air, they are immediately accelerated by local wind, a process requiring support from the momentum of the near-sea wind. Therefore, the wind is slowed down due to the loss of this momentum. The sea spray droplets therefore have the ability to redistribute the momentum in the atmosphere and in the sea water at the air-sea interface.

Andreas $[25,27]$ believed that sea spray momentum flux reached a comparable magnitude to the total air-sea momentum flux at wind speeds above 30-35 m/s, and the air-sea momentum flux was completely converted to sea spray momentum flux when the wind velocity exceeded $60 \mathrm{~m} / \mathrm{s}$. Andreas [25] pointed out that the previously derived high wind speed drag coefficient corresponds to the total momentum flux generated by the wind, and his analysis showed that the sea spray momentum flux modified the total momentum flux generated by the wind. The high wind speed drag coefficient could not be derived from the total momentum flux. Andreas [25] finally concluded that $C_{D}$ stopped increasing and eventually decayed under high wind speed. Researchers had not unified their understanding of the role of droplets in the physics of the air-sea boundary layer. However, it is worth mentioning that Cavaleri et al. [28] believed that droplets make $C_{D}$ begin to decay at high wind speeds. This decay was described by the drag coefficient of both Andreas [25] and Makin [26]. However, when Andreas [25] derived the drag factor at high wind speed, he 
considered the modified action of sea spray momentum flux on the total momentum flux at the air-sea interface, and the sea spray momentum flux representing the foam action was reflected in the high wind speed drag coefficient relation. In the expression of the high wind velocity drag coefficient of Makin [26], he only parametrized the drag coefficient using measurements from Powell [21]. Therefore, the high wind speed drag coefficient relation of Andreas [25] can clearly describe the decay effect on the drag coefficient at high wind speed. Therefore, the theory of Andreas [25] is worth further investigation.

It is worth noting that the generation of sea spray droplets is also related to the wave state [29-32]. As found by the studies mentioned above, when the wave age increases, the number of droplets produced by breaking waves increases as well. However, these studies have not considered the direct effect of sea spray droplets on the air-sea momentum flux. Therefore, under the condition of high wind speeds, the influence of wave state on the total air-sea momentum flux should be considered, as well as the influence of sea spray momentum flux.

The structure of this paper is as follows. Firstly, we provided the expression of the sea surface drag coefficient, which is able to describe the effect on the air-sea momentum flux and includes the sea spray generation function (SSGF) that calculates the sea spray momentum flux. Secondly, we carry out sensitivity experiments, analyzing the influence of wave state on the calculation of sea spray momentum flux. Thirdly, we analyze the influence of wave state on the sea surface drag coefficient and sea surface roughness length in different wave age conditions. Fourthly, we verified the calculated sea surface drag coefficient and sea surface roughness length by using observation data.

\section{Methods}

\subsection{Calculations on Sea Surface Drag Coefficient $\left(C_{D}\right)$}

According to Andreas and DeCosmo [27], the momentum exchange between droplets and the surrounding atmosphere mainly occurs in the evaporative layer of sea spray droplets, which approximately has a thickness of one significant wave height. In the evaporative layer of sea spray droplets, the droplets draw momentum from the air, and the total air-sea momentum flux $\tau_{T}$ can be divided into two parts: the effective air-sea interface momentum flux $\tau_{\text {eff }}$ and the sea spray momentum flux $\tau_{\text {sp. }}$.

$$
\tau_{T}=\tau_{\text {eff }}+\tau_{\mathrm{sp}}
$$

$\tau_{T}$ can generally be obtained by the following formula:

$$
\tau_{T}=\rho_{a} u_{*}^{2}
$$

where $u_{*}$ is the friction velocity, and $\rho_{a}$ is the air density.

The total air-sea momentum flux can also be expressed by the classical bulk formula:

$$
\tau_{T}=\rho_{a} C_{D} U_{10}^{2}
$$

where $C_{D}$ is the drag coefficient that does not exclude the influence of droplets, and $U_{10}$ is the wind speed at $10 \mathrm{~m}$ above the sea surface.

The effective air-sea interface momentum flux $\tau_{\text {eff }}$ is expressed as follows:

$$
\tau_{e f f}=\rho_{a} C_{D, e f f} U_{10}^{2}
$$

where $\tau_{\text {eff }}$ is the effective $C_{D}\left(C_{D, \text { eff }}\right)$ without considering the influence of sea spray droplets.

Substituting Equations (2) and (4) into Equation (1) yields

$$
\rho_{a} C_{D} U_{10}^{2}=\rho_{a} C_{D, e f f} U_{10}^{2}+\tau_{s p}
$$


Then:

$$
C_{D, e f f}=C_{D}-\frac{\tau_{s p}}{\rho_{a} U_{10}^{2}}
$$

Equation (6) is the expression of $C_{D, e f f}$ considering the influence of the droplets.

In Equation (6), it shows that the effective sea surface drag coefficient is affected by the sea spray droplets. The second term on the right is the influence of the sea spray droplets effect. With the increase of the sea spray droplets effect, sea surface drag coefficient is reduced. The sea spray droplet effect is mainly reflected in the ratio of the sea spray momentum flux and sea surface wind speed square. Therefore, accurate estimation of sea spray momentum flux is needed to make good use of Equation (6), to calculate the effective sea surface drag coefficient.

\subsection{Calculations on Sea Spray Momentum Flux}

Sea spray momentum flux $\tau_{s p}$ can be calculated by using Andreas's [25] scheme,

$$
\tau_{s p}=\frac{4 \pi}{3} \rho_{w} \int_{r_{l o}}^{r_{h i}} u_{s p}\left(r_{0}\right) r_{0}^{3} \frac{d F}{d r_{0}} d r_{0}
$$

where $\frac{d F}{d r_{0}}$ is called the SSGF, and $\rho_{w}$ is seawater density. $r_{l o}$ and $r_{h i}$ are the minimum and maximum radii of droplets when calculating $\tau_{s p}$, respectively. $r_{0}$ is the initial radius of the droplet, and $u_{s p}\left(r_{0}\right)$ is the horizontal velocity of the droplet before it falls back to the sea surface. The sea surface wind speed can be expressed as follows:

$$
u_{\mathrm{z}}=\frac{u_{*}}{\kappa} \ln \left(\frac{z}{z_{0}}\right)
$$

Andreas [25] suggested that all droplets be subjected to local winds for a brief flight over the sea surface before falling back. Therefore, $u_{s p}\left(r_{0}\right)$ does not depend on the value of the initial droplet radius, but it depends on the height of the sea spray droplet, which can be expressed as follows:

$$
u_{s p}\left(z_{s}\right)=\frac{u_{*}}{\kappa} \ln \left(\frac{z_{s}}{z_{0}}\right)
$$

where $z_{0}$ is the sea surface roughness length, $\kappa(=0.4)$ is the von Karman constant, and $u_{s p}\left(z_{s}\right)$ is the wind speed at $z_{s}$ above the sea surface. As suggested by Iida et al. [33] and Zhao et al. [29], $z_{s}=0.635 H_{s}$, where $H_{s}$ is the significant wave height, which can be parameterized with the empirical relationship $[14,34]$ :

$$
H_{s}=0.018 U_{10}^{2} \beta^{3 / 2}
$$

where $\beta$ is the wave age parameter, which represents the state of wave growth.

Sea surface roughness length $z_{0}$ can be obtained by using the parameterization scheme of Zhao and Li [4], which depends on the wave state and wind speed:

$$
z_{0}=\left\{\begin{array}{cc}
3.5153 \times 10^{-5} \beta^{-0.42} U_{10}^{2} / g & \text { for } \beta>0.4 \\
2.1583 \times 10^{-4} \beta^{1.56} U_{10}^{2} / g & \text { for } \beta \leq 0.4
\end{array}\right.
$$

\subsection{Sea Spray Generation Function}

In Equation (7), the SSGF $\frac{d F}{d r_{0}}$ is generally regarded as a function of the initial radius $r_{0}$ and $U_{10}$. If the scale spectrum of sea spray droplets is independent of wind speed, the SSGF can be divided into two independent parts: one is only related to wind speed, and the other is only related to droplet size, i.e.,

$$
\frac{d F}{d r_{0}}=f_{1}\left(U_{10}\right) f_{2}\left(r_{0}\right)
$$


where $f_{1}$ and $f_{2}$ are the independent functions of the SSGF.

Andreas $[25,35]$ simplified the sea spray momentum flux and assumed that the sea spray momentum flux was proportional to the fourth power of the friction velocity:

$$
\tau_{s p} \sim u_{*}^{4}
$$

Studies had shown that various functions were related to wind speed among different SSGFs, and there was a considerable difference in magnitude between these functions [28]. In fact, wind is not the only factor that affects the formation of sea spray droplets at the sea surface, as both wind and waves at the surface play important roles. Waves affect the exchanges of air-sea fluxes. Researchers have pointed out that the uncertainty of the calculated value of the sea spray momentum flux was caused by ignoring the influence of the wave state, and they believed that the SSGF should consider not only the influence of wind but also the influence of the wave state [29]. Assuming that the dependence of the SSGF on sea surface wind speed and wave state is independent of the sea spray droplet radius, the SSGF can be divided into two independent functions:

$$
\frac{d F}{d r_{0}}=f_{1}\left(R_{B}\right) f_{2}\left(r_{0}\right)
$$

Here, $R_{B}=\frac{u_{*}^{2}}{\omega_{p} v}$ is the windsea Reynolds number, in which $v$ is the coefficient of air viscosity, and $\omega_{p}$ is the angular frequency corresponding to the peak of the wind wave spectrum. The windsea Reynolds number is widely used in the air-sea boundary layer to study the influence of the wave state on the gas and momentum transports on the sea surface. Together with Equations (2) and (3), $R_{B}$ can also be written as follows:

$$
R_{B}=\frac{U_{10}^{3}}{g v} C_{D} \beta
$$

Applying this theory on the measured data in the laboratory, Zhao et al. [29] proposed a new SSGF:

$$
d F / d r_{0}=\left\{\begin{array}{cc}
7.84 \times 10^{-3} R_{B}^{1.5} r_{0}^{-1}, & 30<r_{0}<75 \mu \mathrm{m} \\
4.41 \times 10^{1} R_{B}^{1.5} r_{0}^{-3}, & 75 \leq r_{0}<200 \mu \mathrm{m} \\
1.41 \times 10^{13} R_{B}^{1.5} r_{0}^{-8}, & 200 \leq r_{0} \leq 500 \mu \mathrm{m}
\end{array}\right.
$$

The calculated results of Equation (16) can well cover the range of the calculated values of the mainstream droplet generation functions [29]. However, this formula is only applicable to sea spray droplets with a radius range of $30 \leq r_{0} \leq 500 \mu \mathrm{m}$, which means that the droplets induced by bubbles (what are their sizes?) are not taken into account.

Based on the coverage rate of whitecaps, Monahan et al. [36] gave the SSGF applicable to the droplets generated by bubbles and the applicable radius of sea spray droplets with a relative humidity of $80 \%$ is $0.8-10 \mu \mathrm{m}$ :

$$
\frac{d F}{d r_{80}}=\frac{W}{\tau_{d}} \frac{d E}{d r_{80}}=W \frac{1}{\tau_{d}} \frac{d E}{d r_{80}}
$$

where $r_{80}$ is the droplet radius when the reference relative humidity is $80 \%, \tau_{d}=3.53 \mathrm{~s}$ is the typical decay time of whitecaps, and $W$ is the whitecap coverage rate. Monahan et al. [36] suggested that $W$ be only related to wind speed. $\frac{d E}{d r_{80}}$ is the number of droplets generated per unit area of whitecap in its decay stage, per unit time and within the increment range of the unit radius corresponding to $r_{80}$.

Monahan et al. [36] noted that $\frac{d E}{d r_{80}}$ can be expressed as follows:

$$
\begin{gathered}
\frac{d E}{d r_{80}}=1.262 \times 10^{6} r_{80}^{-3}\left(1+0.057 r_{80}^{1.05}\right) \times 10^{1.19 \exp \left(-B^{2}\right)} \\
B=\left(0.380-\log r_{80}\right) / 0.650
\end{gathered}
$$


The generation function established in Equation (18) by Monahan et al. [36] has been shown to be useful by several subsequent studies [37-39]. Taking advantage of the tank observation data for whitecaps, Woolf et al. [38] modified the droplet generation function by Monahan et al. [36], which is applicable to bubbles, and pointed out that the revised $\frac{d E}{d r_{80}}$ satisfies

$$
\frac{d E}{d r_{80}}=\exp \left[16.1-3.43 \log r_{80}-2.49\left(\log r_{80}\right)^{2}+1.21\left(\log r_{80}\right)^{3}\right]
$$

The fitting relationships in Equations (18) and (19) are complicated, and the droplet scale spectrum is different from the common spume droplets' spectrum in terms of math expression. In the present study, the least square method is adopted to fit the average values of Equations (18) and (19), yielding $\frac{d E}{d r_{80}}$ with a simpler form. Let $r_{80}$ be in the range of 1-10 $\mu \mathrm{m}$ and substitute into Equations (18) and (19) to calculate the results, then average the results on each $r_{80}$. Based on the average value, the least square method is used to obtain the relationship between $r_{80}$ and $\frac{d E}{d r_{80}}$ :

$$
\frac{d E}{d r_{80}}=5.35 \times 10^{6} r_{80}^{-3} R=0.9995
$$

where $R$ is the correlation coefficient. The following relationship between $r_{80}$ and $r_{0}$ by Andreas [40] is considered:

$$
r_{80}=0.518 r_{0}^{0.976}
$$

Then, the SSGF applicable to droplets with an initial radius of $2 \leq r_{0} \leq 20 \mu \mathrm{m}$ can be expressed as follows:

$$
\frac{d F}{d r_{0}}=f\left(R_{B}\right) r_{0}^{-3}, 2 \leq r_{0} \leq 20 \mu \mathrm{m}
$$

According to Equation (22), the sea spray droplet scale spectrum is simplified as a function of $r_{0}^{-3}$, whereas $f\left(R_{B}\right)$ represents a function related to the wave state. From Zhao et al. [29], we know that the function can be expressed by the wave state parameter $R_{B}$.

Andreas [41] recommended the SSGF based on the data by $\mathrm{Wu}$ [5]:

$$
\frac{d F}{d r_{80}}= \begin{cases}C_{1}\left(U_{10}\right) r_{80}^{-1}, & 10 \leq r_{80} \leq 37.5 \mu \mathrm{m} \\ C_{2}\left(U_{10}\right) r_{80}^{-2.8}, & 37.5 \leq r_{80} \leq 100 \mu \mathrm{m} \\ C_{3}\left(U_{10}\right) r_{80}^{-8}, & 100 \leq r_{80} \leq 250 \mu \mathrm{m}\end{cases}
$$

where $C_{1}, C_{2}$, and $C_{3}$ are functions of $U_{10}$. Based on the calculated value at $U_{10}=15 \mathrm{~m} / \mathrm{s}$ of the SSGF SEA93 proposed by Smith et al. [42], Andreas [41] determined that $C_{1}=1.955 \times 10^{3}$, $C_{2}=1.331 \times 10^{6}$, and $C_{3}=3.344 \times 10^{16}$.

Therefore, based on the sea spray droplet scale spectrum given by Equation (23), the formula by Zhao et al. [29] is revised here. The minimum applicable sea spray droplet radius is extended to $20 \mu \mathrm{m}$, giving:

$$
d F / d r_{0}=\left\{\begin{array}{lc}
7.84 \times 10^{-3} R_{B}^{1.5} r_{0}^{-1}, & 20 \leq r_{0}<75 \mu \mathrm{m} \\
4.41 \times 10^{1} R_{B}^{1.5} r_{0}^{-3}, & 75 \leq r_{0}<200 \mu \mathrm{m} \\
1.41 \times 10^{13} R_{B}^{1.5} r_{0}^{-8}, & 200 \leq r_{0} \leq 500 \mu \mathrm{m}
\end{array}\right.
$$

Considering the continuity of the SSGF, and using Equations (22) and (24), the following relation is obtained:

$$
d F / d r_{0}=\left\{\begin{array}{lc}
3.14 \times 10^{0} R_{B}^{1.5} r_{0}^{-3}, & 2 \leq r_{0}<20 \mu \mathrm{m} \\
7.84 \times 10^{-3} R_{B}^{1.5} r_{0}^{-1}, & 20 \leq r_{0}<75 \mu \mathrm{m} \\
4.41 \times 10^{1} R_{B}^{1.5} r_{0}^{-3}, & 75 \leq r_{0}<200 \mu \mathrm{m} \\
1.41 \times 10^{13} R_{B}^{1.5} r_{0}^{-8}, & 200 \leq r_{0} \leq 500 \mu \mathrm{m}
\end{array}\right.
$$


According to Equation (25), $d F / d r_{0}$ is an expression composed of four sections, and its value is proportional to $R_{B}$ and inversely proportional to $r_{0}$. The variation trend of spray droplet number with $r_{0}$ in the first section is different from that in the other three sections. In the first section, $d F / d r_{0}$ is proportional to $r_{0}^{-3}$, and the other three sections are mainly in a similar form to $\mathrm{Wu}[5]$, that is, $d F / d r_{0}$ is proportional to $r_{0}^{-1}, r_{0}^{-3}$, and $r_{0}^{-8}$ as the range of radius increases. This is because the first section describes sea spray droplets produced by bubbles, and its generation mechanism is different from that described in the other three sections.

With Equations (7), (9)-(11), and (25), the momentum flux of sea spray droplets with a radius from 2 to $500 \mu \mathrm{m}$ can be calculated, which incorporates the influence of the wave state.

\section{Results}

\subsection{Wave State Influence on Sea Spray Momentum Flux}

From Equation (7), we can see that the core of sea spray momentum flux is the SSGF. Most of the existing sea spray generation functions do not consider the influence of waves. As shown in Figure 1, the sea spray generation function in Equation (25) has different values under different wave ages, which confirms that it is necessary to consider the effect of the wave state in the sea spray generation function. Note that the sea spray generation function increases with the increase of wave age, instead of showing a varied trend as a function of wave age, as indicated in Equation (11). This is due to the fact that the significant wave height in Equation (10) increases with the increase of wave age. Therefore, the sea spray generation function maintains an increasing trend when wave age increases, though with a faster growth when the wave age is smaller than 0.4 and a slower increase when wave age is larger than 0.4 .

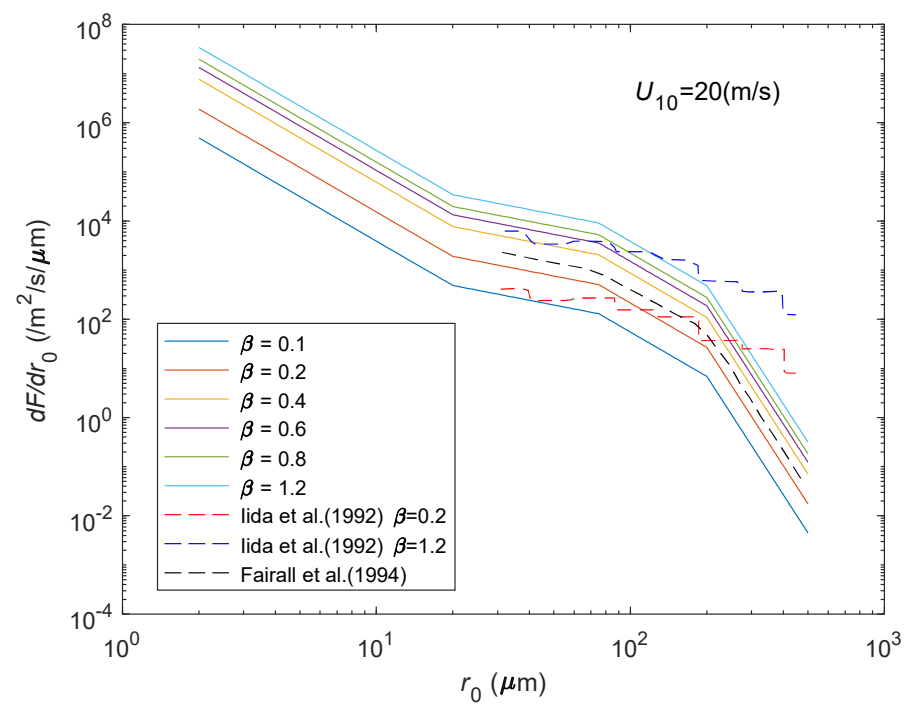

Figure 1. Distribution of the sea spray generation function under different wave ages.

The droplets accelerated by wind extract momentum from the wind near the sea surface and slow down the wind speed. Figure 2 shows the variation of the ratio of sea spray momentum flux to the total momentum flux, with wind speed at different wave ages. We can see that when the wind speed is fixed, with the growth of the wave, the ratio increases, which further indicates that the wave state has a great impact on the generation of the sea spray momentum flux. As shown in Figure 2, the sea spray momentum flux can exceed the order of $10^{1}$ in different wave growth states, which increases with the growth of waves when the wind speed is constant. The variation of the total momentum flux with wind speed is displayed in Figure 2. When the wave age is varied, the variation of the total momentum flux is not as obvious as that of the sea spray momentum flux. When the wind 
speed reaches approximately $35-80 \mathrm{~m} / \mathrm{s}$, the sea spray momentum flux can reach the order of the total momentum flux under different wave age conditions. Therefore, it is known that the wave state has a great influence on the order of the sea spray momentum flux. The larger the wave age is, the earlier the momentum flux of the sea spray droplet reaches the same order as the total momentum flux.

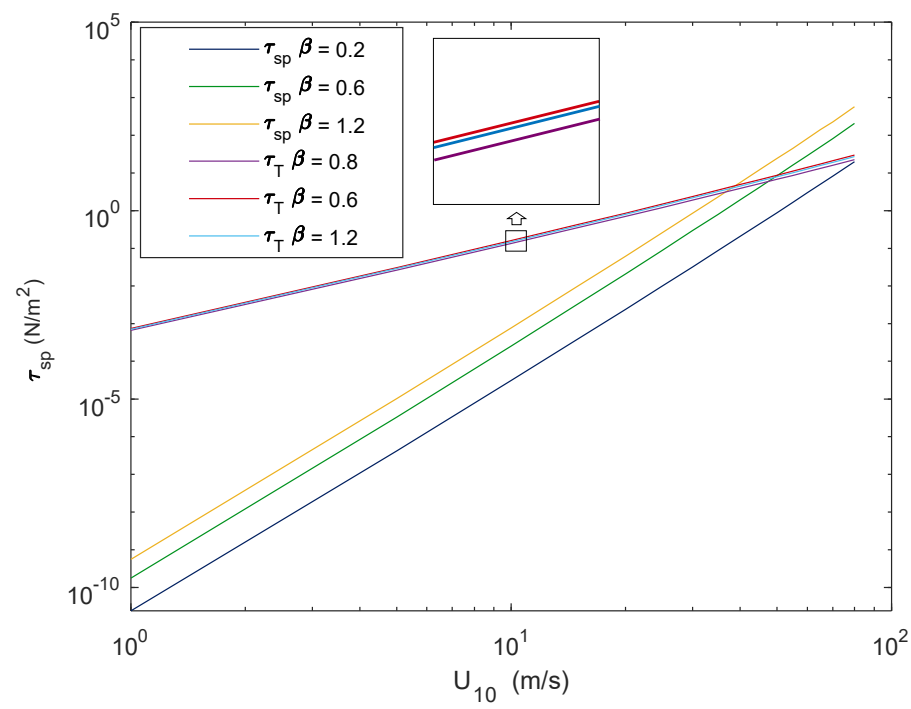

Figure 2. Variations of the spray momentum flux and total momentum flux, as a function of wind speed under different wave ages.

Since the sea spray momentum flux is related to the wave state, the wave state parameters $R_{B}$ can be selected for parameterization of the wave state [29]. $R_{B}$ is the wave breaking control parameter of the air-sea interface, which can express the joint impact of waves and wind on air-sea flux. Figure 3 shows the variation of the sea spray momentum flux as a function of $R_{B}$ under different wave ages. Different from Figure 2, the change of the sea spray droplet momentum flux under different wave states is small here. This means that, compared to the parameterization of the sea spray droplet momentum flux only by wind speed, $R_{B}$ can better fit the variation of the sea spray droplet momentum flux under various wave ages.

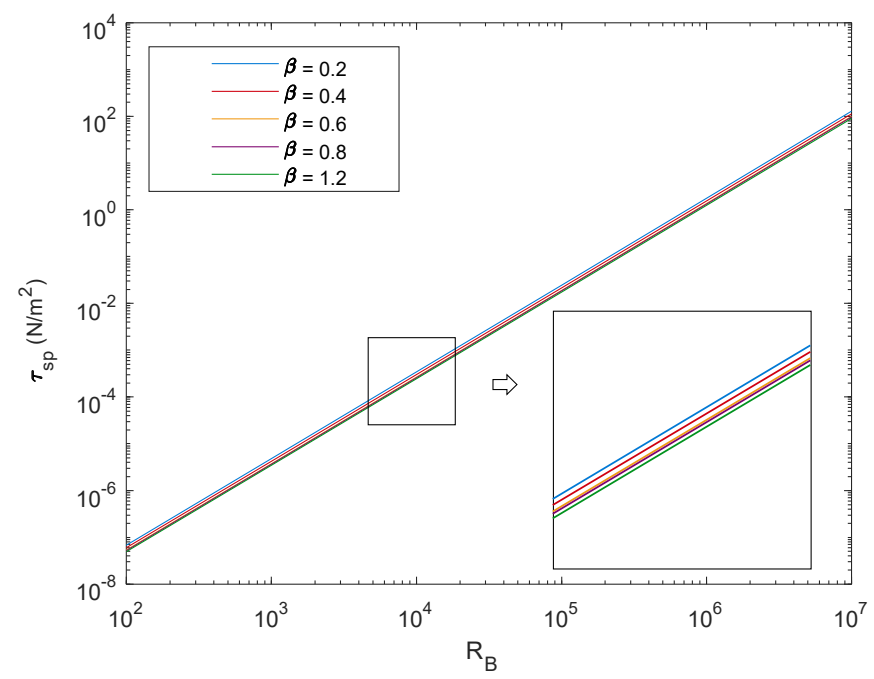

Figure 3. Variations of the sea spray droplet momentum flux, as a function of $R_{B}$ under different wave ages. 
Figure 4 also shows the variation of the droplet momentum flux to the total momentum flux ratio under different wave ages. When the value of $R_{B}$ reaches $2 \times 10^{6}$, the ratio is close to one, which indicates that the two fluxes are of the same order of magnitude. When the value of $R_{B}$ is fixed, the flux ratio increases with the growth of the waves.

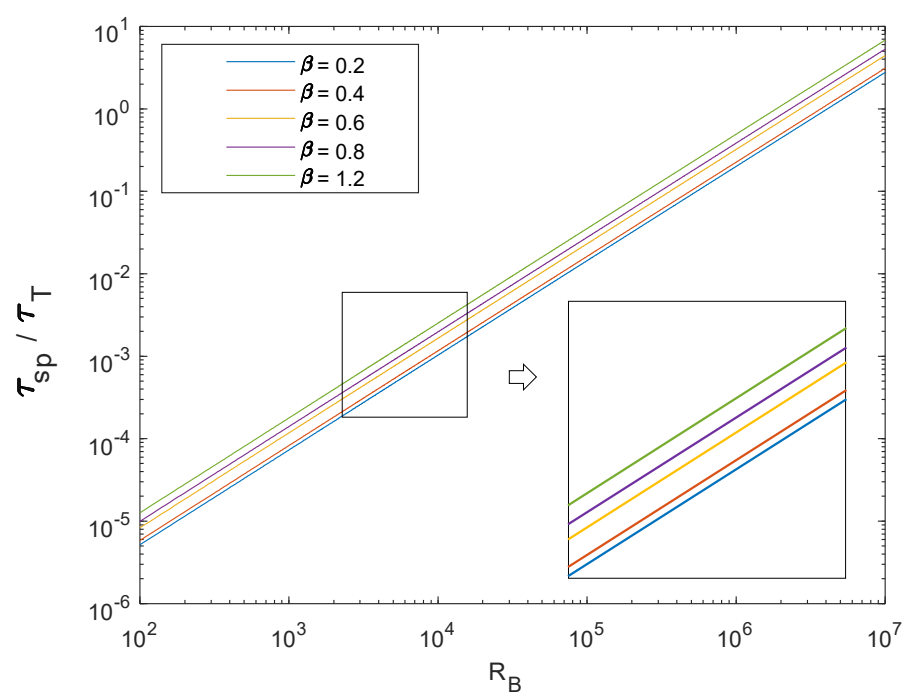

Figure 4. Variations of the sea spray droplet momentum flux to the total momentum flux ratio, as a function of $R_{B}$ under different wave ages.

The above analysis shows that the windsea Reynolds number $R_{B}$ can fit the sea spray droplet momentum flux well under various wave conditions. After transformation, the relationship between $R_{B}$, friction velocity, and wave age becomes:

$$
R_{B}=\frac{U_{10}^{3}}{g v} C_{D} \beta=\frac{u_{*}^{3}}{g v C_{D}^{1 / 2}} \beta
$$

When the friction velocity and wave age are fixed, the value of $C_{D}$ affects $R_{B}$ as well.

Andreas $[25,35]$ simplified the sea spray droplet momentum flux by considering that the sea surface droplet stress to be related to the fourth power of the friction velocity, i.e.,

$$
\tau_{s p}=6.2 \times 10^{-5} \rho_{w} u_{*}^{4}
$$

The results from the formula above are compared with the results of Equation (25) proposed in this study (as shown in Figure 5). The average of $R_{B}$ is taken for estimation, and the results are shown in Figure 5. We can see that the sea spray droplets calculated by Equation (25) can cover those by Equation (27) under different wave ages. This not only demonstrates the correctness of Equation (25), but also shows that the wave state has an impact on the sea spray droplet momentum flux, which should be considered when calculating the droplet momentum flux.

\subsection{Sea Surface Drag Coefficient}

The relationship between sea surface roughness $z_{0}$ and $C_{D}$ can be obtained by combining Equations (2), (3), and (8):

$$
C_{D}=\left(\frac{\kappa}{\ln \left(10 / z_{0}\right)}\right)^{2}
$$

Therefore, $C_{D}$ is related to wave age $\beta$ and surface wind speed at $10 \mathrm{~m}\left(U_{10}\right)$. Figure 6 displays the variation of $C_{D}$ with wind speed under different wave ages without considering the influence of sea spray droplets. When the wave age is less than $0.4, C_{D}$ increases with the increase of wave age. When the wave age is greater than $0.4, C_{D}$ decreases with 
the increase of wave age. The drag coefficient variation with wave age is identical to that of $z_{0}$. When wind speed is less than $30 \mathrm{~m} / \mathrm{s}$, the calculated value of $C_{D}$ covers the result of $\mathrm{Wu}$ [5], which indicates that Equation (28) is correct at low or medium wind speeds. When the wind speed increases, the result becomes smaller than that of $\mathrm{Wu}$ [5]. The $C_{D}$ in $\mathrm{Wu}$ [5] increased linearly with wind speed, which overestimated $C_{D}$ at high wind speeds, as recent studies have shown that $C_{D}$ decays at high wind speeds. To clearly show the wind speed scenarios when the attenuation of $C_{D}$ occurs, Equation (6) is analyzed, considering the influence of droplets on $C_{D}$ under high wind speeds.

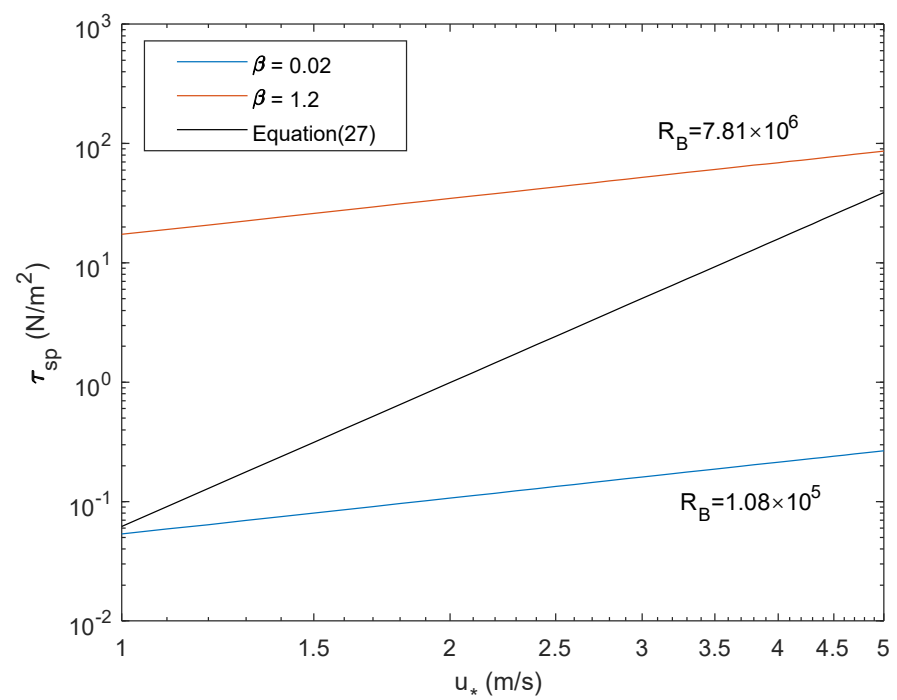

Figure 5. Comparison of the sea spray momentum flux and the result from Equation (27) under different wave ages.

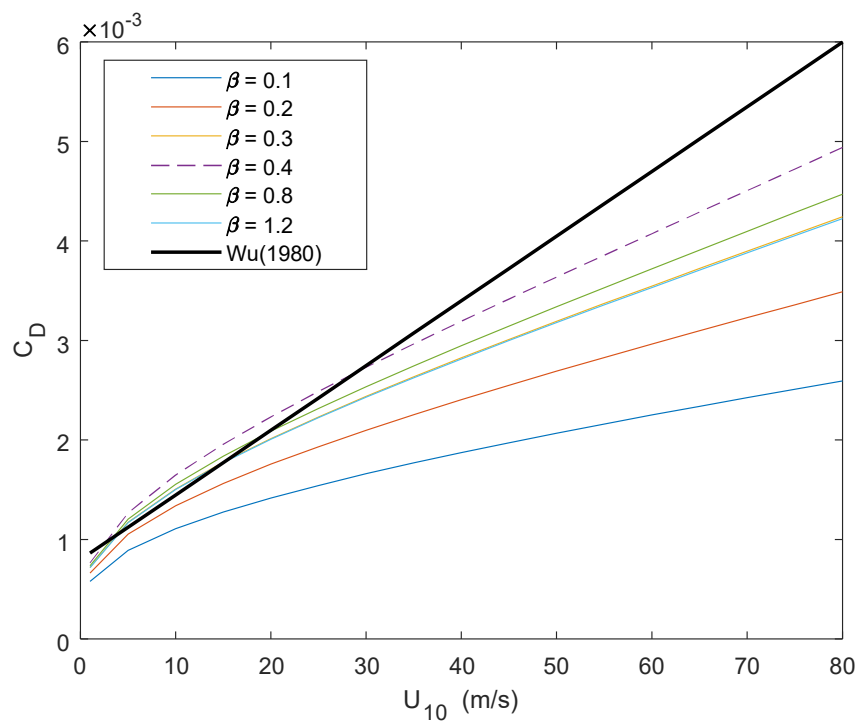

Figure 6. Variations of the sea surface drag coefficient with wind speed under different wave ages, without considering the impact of sea spray droplets. The dashed line $(\beta=0.4)$ indicates that the variation trend of the sea surface drag coefficient with wave age began to change.

Based on Equation (6), the variation of $C_{D}$ with the presence of sea spray droplets can be analyzed. In other words, $C_{D}$ now becomes the effective $C_{D}$, namely $C_{D, e f f}$. Figure 7 shows that when wind speed is greater than $20 \mathrm{~m} / \mathrm{s}, C_{D, e f f}$ is somewhat attenuated, but the corresponding initial attenuation wind speeds differ remarkably for different wave ages. For example, when the wave age is $0.2, C_{D, e f f}$ starts its attenuation at a wind speed of about $50 \mathrm{~m} / \mathrm{s}$. When the wave age is 1.2 , however, the attenuation of $C_{D, e f f}$ occurs 
at a wind speed of $20 \mathrm{~m} / \mathrm{s}$, which indicates that the attenuation of $C_{D, e f f}$ is affected by the wave state. When wind speed is higher than $45 \mathrm{~m} / \mathrm{s}$, the drag coefficient tends to be larger when the wave age is less than 0.4 than when the wave age is greater than 0.4. $C_{D}$ under different wave ages can basically reproduce the high-wind-speed offshore observation data of Powell et al. [21] and Jarosz et al. [43] and the laboratory observation data of Donelan et al. [23] and Takagaki et al. [44].

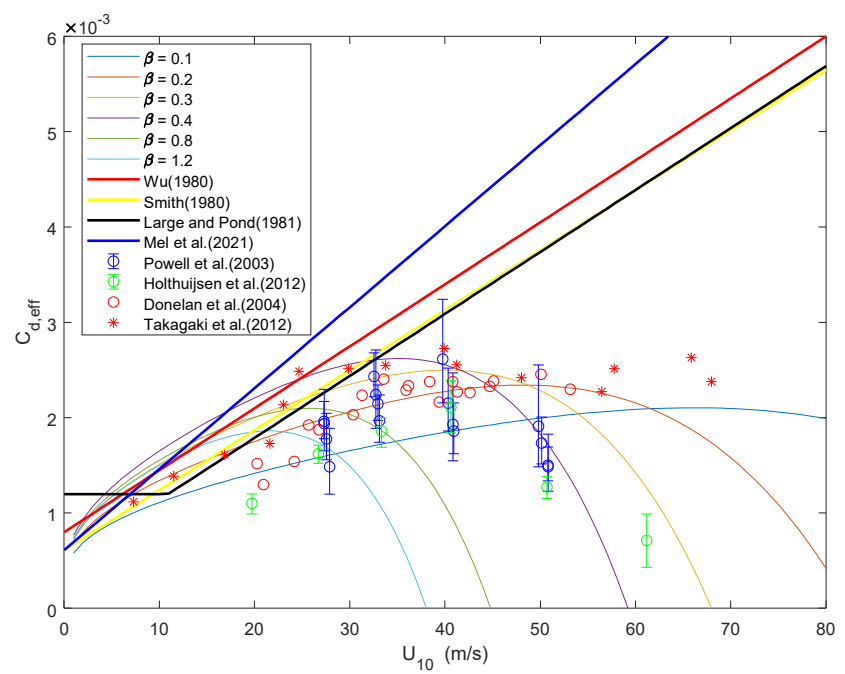

Figure 7. Variations of the effective sea surface drag coefficient with wind speed, considering the impact of sea spray droplets.

In the laboratory, the fetch length is short, so the ocean waves have a small wave age. Meanwhile, the ocean waves have the bigger wave age in the offshore. Especially, in the typhoon condition, because of the change of the wind direction, the waves are difficult to fully grow. Therefore, we use the small wave age calculated results to analyze the errors with the laboratory observation data and use the medium wave age calculation results to analyze the errors with the offshore observation data, as shown in Table 1 . As can be seen from the root mean square error, the error of Equation (6) in the laboratory is smaller than that in the offshore. However, it is much better than the result of the Large and Pond (1981) scheme. This analysis indicates that the influence of sea spray droplets and wave state on the sea surface drag coefficient should be considered under high wind speed conditions.

Table 1. Error between calculated results and observed data with different wave age.

\begin{tabular}{|c|c|c|c|c|c|}
\hline \multicolumn{3}{|c|}{ Laboratory Observation Data } & \multicolumn{3}{|c|}{ Offshore Observation Data } \\
\hline \multirow[b]{2}{*}{ Wave Age } & \multicolumn{2}{|c|}{ Root-Mean-Square Error } & \multirow[b]{2}{*}{ Wave Age } & \multicolumn{2}{|c|}{ Root-Mean-Square Error } \\
\hline & Equation (6) & $\begin{array}{c}\text { Large and } \\
\text { Pond (1981) }\end{array}$ & & Equation (6) & $\begin{array}{c}\text { Large and } \\
\text { Pond (1981) }\end{array}$ \\
\hline 0.1 & $4.41 \times 10^{-4}$ & \multirow{2}{*}{$7.32 \times 10^{-4}$} & 0.5 & $4.92 \times 10^{-4}$ & \multirow{2}{*}{$1.20 \times 10^{-3}$} \\
\hline 0.2 & $2.01 \times 10^{-4}$ & & 0.6 & $6.68 \times 10^{-4}$ & \\
\hline
\end{tabular}

\subsection{Sea Surface Roughness}

There is a one-to-one relationship between $C_{D}$ and sea surface roughness. Equation (11) states that sea surface roughness increases with the increase of wind speed. As shown in Figure 8, when the wave age is less than 0.4, the sea surface roughness increases with the increase of wave age. However, when the wave age is greater than 0.4 , sea surface roughness decreases with the increase of wave age. 


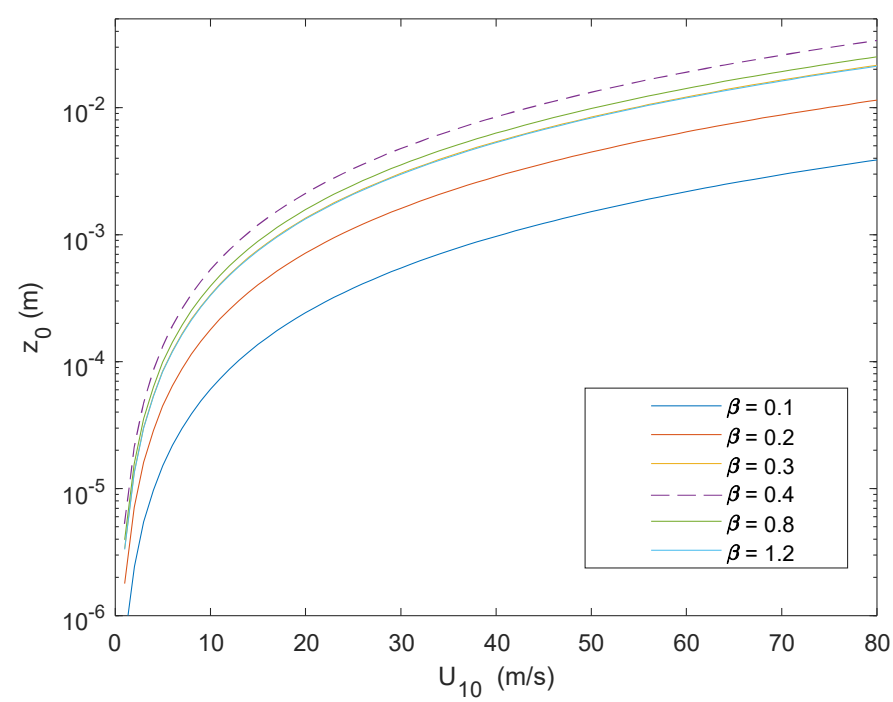

Figure 8. Variations of the sea surface drag coefficient with wind speed under different wave ages, without considering the impact of sea spray droplets. The dashed line $(\beta=0.4)$ indicates that the variation trend of sea surface roughness with wave age began to change.

Similarly, using Equation (26), the effective sea surface roughness $\left(z_{0, e f f}\right)$ corresponding to $C_{D, e f f}$ can be obtained as follows:

$$
z_{0, e f f}=10 e^{\frac{-\kappa}{\sqrt{C_{D, e f f}}}}
$$

According to Figure 9, sea surface roughness also decays with the increase of wind speed, and the wind speed, at which the roughness decays, varies at different wave ages. Compared with the high wind speed offshore observation data by Powell et al. [21] and the laboratory observation data by Takagaki et al. [44], Equation (29) can well reproduce the measured values under different wave ages, with a variation trend similar to that of the measured values.

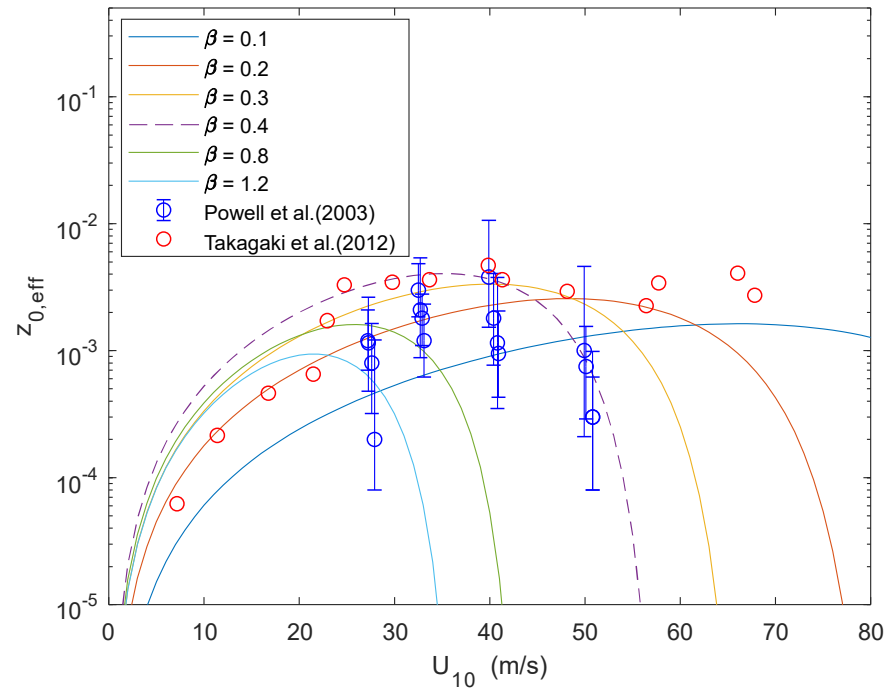

Figure 9. Variations of effective sea surface roughness with wind speed, considering the impact of sea spray droplets.

\section{Discussion}

To further analyze the influence of wave age on $C_{D}$ in detail, the range of wave age is selected as 0.01 to 1.20 . When the wave age is smaller than 0.4 and wind speed is low, $C_{D}$ 
increases with the increase of wave age, as shown by the blue shading of low wind speed in Figure 10. With the increase of wind speed, this relationship is reversed, that is, the drag coefficient decreases with the increase of wave age. This is because the momentum flux of sea spray droplets keeps increasing with the increase of wave age (shown in red in Figure 2), i.e., the momentum flux acquired from the air increases with the increase of wind speed at high wind speeds. When the wave age is greater than 0.4 but the wind speed is changing from low-to-medium to high, $C_{D, e f f}$ decreases with the increase of wave age from low to high wind speeds (as shown by the blue shading of Figure 10). In this case, the sea spray droplet momentum flux increases with the increase of wave age, which highlights the relation that $C_{D, e f f}$ decreases with the increase of wave age.

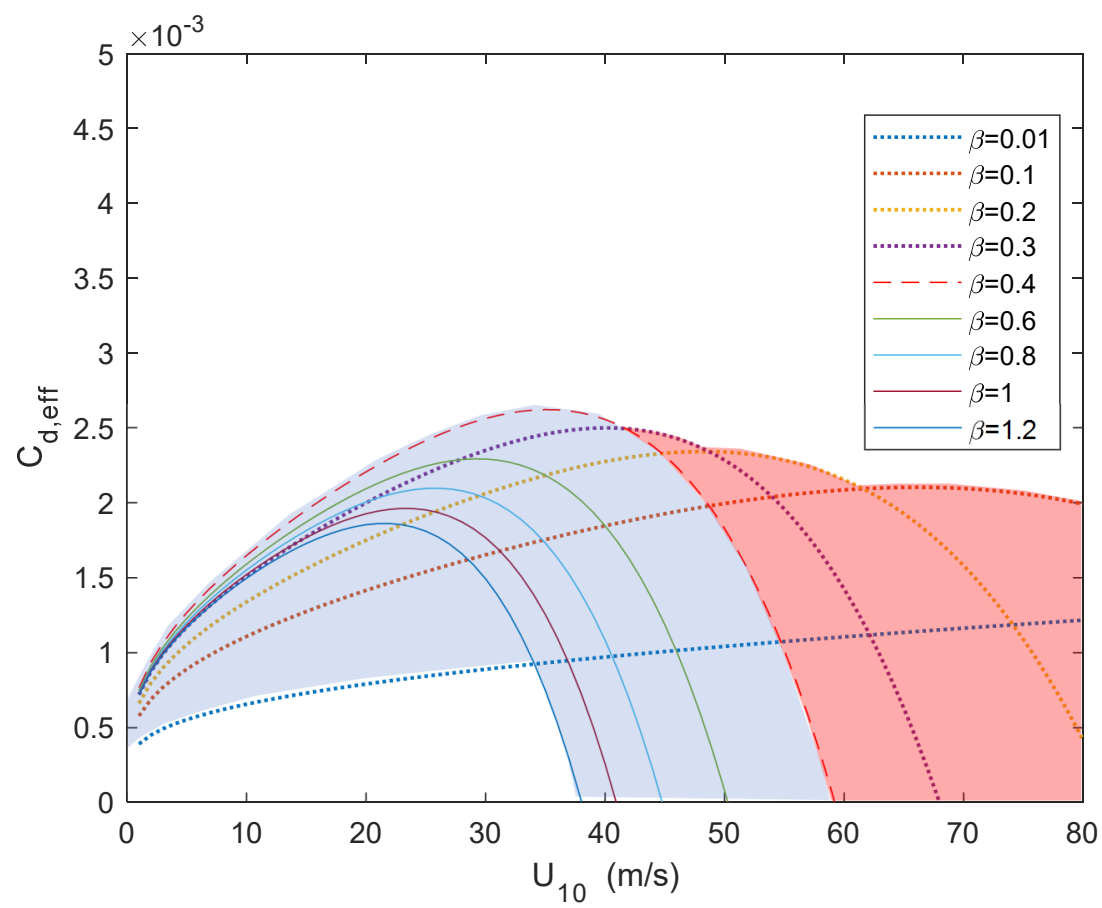

Figure 10. Influence of wave state on the drag coefficient.

Through the comparison of Figures 7 and 9, it can be seen that there are some differences between the offshore measurement data and the laboratory measurement data, in terms of the variation trend and range as a function of wind speed. When the wind speed is low to medium, $C_{D, e f f}$ and effective sea surface roughness in the open sea are lower than those measured in the laboratory. Holthuijsen et al. [22] stated that sea surface roughness was reduced due to the effect of a storm surge.

It is worth noting that in some wave states, $C_{D}$ may decrease to zero with the increase of wind speed, as shown in Figure 10. This phenomenon also appeared in Andreas [25]. However, when $C_{D}$ becomes zero, it means that the momentum of the atmosphere will not be directly fed into the ocean. Andreas [25] reported that when sea spray droplets returned to the sea surface, the short waves of the sea surface would disappear, thus inhibiting the growth of waves. In this scenario, it is difficult for the wave to grow sufficiently. In other words, it is not easy to see the drag coefficient corresponding to larger wave ages in Figure 10 when it is approaching zero.

When wind speed is greater than $50 \mathrm{~m} / \mathrm{s}$, the $C_{D}$ measured in the laboratory is significantly greater than that measured in the open sea. As shown in Figure 7, Donelan et al. [23] stated that the air flow would separate from the wave surface and jump directly from the crest of one breaking wave to another crest, without passing through the trough. The existence of these continuous breaking waves would restrict the growth of wind waves, reducing $C_{D}$. In the open sea, the above-stated process holds as well. In addition, according to Holthuijsen et al. [22], when wind speed is high, a large number of sea spray droplets is 
generated in the open sea, forming a layer of sea spray droplets, which lowers sea surface roughness. As the wave height and wavelength in the tank are smaller than those in the open sea (see Donelan et al. [23], Figure 4), the droplets would return to the sea surface soon, making it difficult to form a spray droplet layer. Therefore, the reduction of the drag coefficient in the tank is smaller than that in the open sea.

According to the analysis results of open-sea and laboratory measurement data in Zhao et al. [4], the wave age in the open-sea measurement data is generally greater than 0.4 , while the wave age in the laboratory measurement data is typically less than 0.4. Although these were measured under low and medium wind speeds, it also shows that there are differences in wave age between the laboratory tank and the open sea. Using the $C_{D}$ at high wind speeds discussed in this study (Figure 7), we see that when the wind speed is higher than $50 \mathrm{~m} / \mathrm{s}$, the laboratory measurement data are mainly distributed in the region with low wave ages. Therefore, it can be inferred that the wave age in the tank is still small at high wind speeds.

\section{Conclusions}

At high wind speeds, sea spray droplets are generated by wave breaking. The sea surface drag coefficient stops increasing and decays when the wind speed reaches a certain level. Although this phenomenon has been confirmed by observations in the open sea and laboratories, there still exist some controversies about the physical mechanism of this attenuation. In this paper, a new relationship between the sea surface drag coefficient and wave state is constructed from the influence of wave state on the drag coefficient and the influence of wave state on the generation of sea spray droplets.

Firstly, we establish an SSGF (Equation (25)), which can further calculate the momentum flux of sea spray droplets with a radius from 2 to $500 \mu \mathrm{m}$. We show that sea spray droplets absorb the wind energy above the sea surface, which produces the sea spray momentum flux. The sea spray momentum flux increases with the increase of wind speed and wave age, which attenuate the effective momentum actually transmitted at the air-sea interface.

Secondly, based on a new spray droplet generation function (Equation (25)), sea spray momentum flux can be calculated, and a new sea surface drag coefficient under high wind speeds is established (Equation (6)), which takes into account the influence of sea spray droplets and wave state. Compared with the sea surface drag coefficient at medium wind speeds, we find that Equation (6) also performs well in these cases. According to the calculation, the effective sea surface drag coefficient at high wind speeds is attenuated, and the attenuation is different under different wave age conditions. Comparing the observation data in the open sea and from the laboratory at high wind speeds, we find that the calculated drag coefficient under different wave age conditions can basically cover the measured values. Therefore, the sea surface drag coefficient given by Equation (6) under high wind speeds is recommended for calculating air-sea momentum fluxes in the open sea and laboratory tanks.

In the future, with the increase of observation data under high wind speeds, especially for sea spray droplets, the SSGF will be more reasonable, and the calculation accuracy of sea spray momentum flux can be further improved. At the same time, the influence of ocean current on the sea surface drag coefficient can be considered in the parameterization scheme, which can further improve the theory of air-sea interaction and provide an accurate airsea momentum flux for simulating marine environments in ocean-atmosphere coupling models under high wind speeds.

Author Contributions: Conceptualization, J.S. and Y.S.; data curation, J.S. and Z.F.; formal analysis, J.S., Z.F., Y.S. and X.Z.; funding acquisition, Y.S.; investigation, J.S., W.Z. and Y.Y.; visualization, J.S.; writing —original draft, J.S. and Z.F.; writing—review and editing, Y.S. All authors have read and agreed to the published version of the manuscript.

Funding: This work is supported by the National Natural Science Foundation of China (No. 42075035 and 41676014). 
Institutional Review Board Statement: Not applicable.

Informed Consent Statement: Not applicable.

Data Availability Statement: The reader can ask for all the related data from the first author (shijian@nudt.edu.cn) and the corresponding author (sunyuan17a@nudt.edu.cn).

Acknowledgments: The authors would like to thank C.W. Fairall for his observational data in the laboratory.

Conflicts of Interest: The authors declare no conflict of interest.

\section{References}

1. Charnock, H. Wind stress on a water surface. Q. J. R. Meteorol. Soc. 1955, 81, 639-640. [CrossRef]

2. Lafon, C.; Piazzola, J.; Forget, P.; Le Calve, O.; Despiau, S. Analysis of the Variation of the Whitecap Fraction as Measured in a Coastalzone. Bound. Layer Meteorol. 2004, 111, 339-360.

3. Guan, C.; Xie, L. On the Linear Parameterization of Drag Coefcient over Sea Surface. J. Phys. Oceanogr. 2004, 34, 847-2851.

4. Zhao, D.; Li, M. Dependence of Wind Stress across an Air-sea Interface on Wave States. J. Oceanogr. 2019, 75, $207-223$.

5. Wu, J. Wind-stress Coefcients over Sea Surface near Neutral Conditions-A Revisit. J. Phys. Oceanogr. 1980, 10, 727-740.

6. Large, W.G.; Pond, S. Open Ocean Momentum Fux Measurements in Moderate to Strong Winds. J. Phys. Oceanogr. 1981, 11, 324-336.

7. Geernaert, G.L.; Larsen, S.E.; Hansen, F. Measurements of the wind stress, heat flux, and turbulence intensity during storm conditions over the North Sea. J. Geophys. Res. Space Phys. 1987, 92, 13127-13139. [CrossRef]

8. Yelland, M.; Taylor, P.K. Wind Stress Measurements from the Open Ocean. J. Phys. Oceanogr. 1996, 26, 541-558. [CrossRef]

9. Borkowski, P. Numerical Modeling of Wave Disturbances in the Process of Ship Movement Control. Algorithms 2018, 11, 130. [CrossRef]

10. Murashige, S.; Choi, W. A numerical study on parasitic capillary waves using unsteady conformal mapping. J. Comput. Phys. 2017, 328, 234-257. [CrossRef]

11. Donelan, M.A.; Le Méhauté, B.; Hanes, D.M. Air-Sea Interaction. The Sea: Ocean Engineering Science. J. Wiley. 1990, 9, $239-292$.

12. Toba, Y.; Iida, N.; Kawamura, H.; Ebuchi, N.; Jones, I.S.F. Wave Dependence of Sea-Surface Wind Stress. J. Phys. Oceanogr. 1990, 20, 705-721. [CrossRef]

13. Shi, J.; Zhong, Z.; Li, R.; Li, Y.; Sha, W. Dependence of sea surface drag coefficient on wind-wave parameters. Acta Oceanol. Sin. 2011, 30, 14-24. [CrossRef]

14. Toba, Y. Local balance in the air-sea boundary processes. J. Oceanogr. 1972, 28, 109-120. [CrossRef]

15. Janssen, P.A.E.M. Wave-Induced Stress and the Drag of Air Flow over Sea Waves. J. Phys. Oceanogr. 1989, 19, 745-754. [CrossRef]

16. Jiménez, P.A.; Dudhia, J. On the Need to Modify the Sea Surface Roughness Formulation over Shallow Waters. J. Appl. Meteorol. Clim. 2018, 57, 1101-1110. [CrossRef]

17. Mel, R.A.; Viero, D.P.; Carniello, L.; Defina, A.; D'Alpaos, L. The first operations of Mo.S.E. system to prevent the flooding of Venice: Insights on the hydrodynamics of a regulated lagoon. Estuar. Coast. Shelf Sci. 2021, 261, 107547. [CrossRef]

18. Hsu, S.A. A Mechanism for the Increase of Wind Stress (Drag) Coefficient with Wind Speed over Water Surfaces: A Parametric Model. J. Phys. Oceanogr. 1986, 16, 144-150.

19. Nystrom, R.G.; Rotunno, R.; Davis, C.A.; Zhang, F. Consistent Impacts of Surface Enthalpy and Drag Coefficient Uncertainty between an Analytical Model and Simulated Tropical Cyclone Maximum Intensity and Storm Structure. J. Atmos. Sci. 2020, 77, 3059-3080.

20. Moon, I.; Ginis, I.; Hara, T. Impact of the Reduced Drag Coefficient on Ocean Wave Modeling under Hurricane Conditions. Mon. Weather Rev. 2008, 136, 1217-1223.

21. Powell, M.D.; Vickery, P.J.; Reinhold, T.A. Reduced drag coefficient for high wind speeds in tropical cyclones. Nat. Cell Biol. 2003, 422, 279-283. [CrossRef]

22. Holthuijsen, L.H.; Powell, M.; Pietrzak, J.D. Wind and waves in extreme hurricanes. J. Geophys. Res. Space Phys. 2012, 117, 09003 [CrossRef]

23. Donelan, M.A.; Haus, B.K.; Reul, N.; Plant, W.J.; Stiassnie, M.; Graber, H.C.; Brown, O.; Saltzman, E.S. On the limiting aerodynamic roughness of the ocean in very strong winds. Geophys. Res. Lett. 2004, 31, L18306. [CrossRef]

24. Takagaki, N.; Komori, S.; Suzuki, N. Estimation of friction velocity from the wind-wave spectrum at extremely high wind speeds. In IOP Conference Series: Earth and Environmental Science; IOP Publishing: Bristol, UK, 2016; Volume 35, p. 012009.

25. Andreas, E.L. Spray Stress Revisited. J. Phys. Oceanogr. 2004, 34, 1429-1440. [CrossRef]

26. Makin, V.K. A Note on the Drag of the Sea Surface at Hurricane Winds. Bound. Layer Meteorol. 2005, 115, 169-176. [CrossRef]

27. Andreas, E.L.; DeCosmo, J. The Signature of Sea Spray in the HEXOS Turbulent Heat FluxData. Bound. Layer Meteor. 2002, 103, 303-333.

28. Cavaleri, L. Wave Modeling-The State of the Art. Prog. Oceanogr. 2007, 75, 603-674.

29. Zhao, D.; Toba, Y.; Sugioka, K.-I.; Komori, S. New sea spray generation function for spume droplets. J. Geophys. Res. Space Phys. 2006, 111, 02007. [CrossRef] 
30. Shi, J.; Zhao, D.L.; Li, X.Q.; Zhong, Z. New Wave Dependent Formulae for Sea Spray Flux. J. Hydrodyn. 2009, $21,573-581$.

31. Shi, J.; Zhong, Z.; Li, X.; Jiang, G.; Zeng, W.; Li, Y. The Influence of wave state and sea spray on drag coefficient from low to high wind speeds. J. Ocean Univ. China 2016, 15, 41-49. [CrossRef]

32. Wan, Z.-H.; Zhu, J.-B.; Sun, K.; Zhou, K. An integrated turbulent simulation and parameter modeling study on sea-spray dynamics and fluxes. Ocean Eng. 2017, 130, 64-71. [CrossRef]

33. Iida, N.; Toba, Y.; Chaen, M. A new expression for the production rate of sea water droplets on the sea surface. J. Oceanogr. 1992, 48, 439-460. [CrossRef]

34. Hanson, J.L.; Phillips, O.M. Wind Sea Growth and Dissipation in the Open Ocean. J. Phys. Oceanogr. 1999, 29, $1633-1648$.

35. Andreas, E.L.; Emanuel, K.A. Effects of Sea Spray on Tropical Cyclone Intensity. J. Atmos. Sci. 2001, 58, 3741-3751.

36. Monahan, E.C.; Spiel, D.E.; Davidson, K.L. A model of Marine Aerosol Generation Via Whitecaps and Wave Disruption. In Oceanic Whitecaps and Their Role in Air-Sea Exchange Processes; Monahan, E.C., Niocaill, M.G., Eds.; Reidel: Dordrecht, The Netherlands, 1986; pp. 167-174.

37. Stramska, M. Vertical Profiles of Sea-Salt Aerosol in the Atmospheric Surface Layer: A Numerical Model. ACTA Geophys. Polonica 1987, 35, 87-100.

38. Woolf, D.K.; Monahan, E.C.; Spiel, D.E. Quantification of the Marine Aerosol Produced by Whitecaps. In Proceedings of the 7th Congress on Ocean-Atmosphere Interaction, Anaheim, CA, USA, 31 January-5 February 1988; Preprint Volume, pp. $182-185$.

39. Gong, S.L. A parameterization of sea-salt aerosol source function for sub- and super-micron particles. Glob. Biogeochem. Cycles 2003, 17, 1097. [CrossRef]

40. Andreas, E.L. Sea Spray and the Turbulent Air-Sea Heat Fluxes. J. Geophys. Res. Space Phys. 1992, 97, 11429-11441. [CrossRef]

41. Andreas, E.L. A New Sea Spray Generation Function for Wind Speeds up to $32 \mathrm{~m} \mathrm{~s}^{-1}$. J. Phys. Oceanogr. 1998, 28, 2175-2184. [CrossRef]

42. Smith, M.H.; Park, P.M.; Consterdine, I.E. Marine Aerosol Concentrations and Estimated Fluxes over the Sea. Q. J. R. Meteorol. Soc. 1993, 119, 809-824.

43. Jarosz, E.; Mitchell, D.A.; Wang, D.W.; Teague, W.J. Bottom-Up Determination of Air-Sea Momentum Exchange Under a Major Tropical Cyclone. Science 2007, 315, 1707-1709. [CrossRef]

44. Takagaki, N.; Komori, S.; Suzuki, N.; Iwano, K.; Kuramoto, T.; Shimada, S.; Kurose, R.; Takahashi, K. Strong Correlation between the Drag Coefcient and the Shape of the Wind Sea Spectrum over a Broad Range of Wind Speeds. Geophys. Res. Lett. 2012, 39 , L23604. 